\title{
Macrófitas aquáticas de um rio temporário no semiárido nordestino
}

\section{Aquatic macrophytes of a temporary river in the northeastern semi-arid region}

\begin{abstract}
Macrófitos acuático un río temporal en el noreste semiárido
\end{abstract}

Maria Rosiane Batista dos Santos ${ }^{1}$

Maria Carolina de Abreu ${ }^{2}$

Ana Paula Peron ${ }^{3}$

Maria do Socorro Meireles de Deus ${ }^{4}$

${ }^{1}$ Graduanda do curso de Ciências Biológicas na Universidade Federal do Piauí, Picos, PI. E-mail: biorosiane@hotmail.com

${ }^{2}$ Professora do curso de Ciências Biológicas da Universidade Federal do Piauí, Picos, PI. Doutora em Botânica pela Universidade Federal Rural de Pernambuco (UFRPE). E-mail: mariacarolinabreu@hotmail.com

${ }^{3}$ Professora do curso de Ciências Biológicas da Universidade Federal do Piauí, Picos, PI. Doutora em Ciências Ambientais pela Universidade Estadual de Maringá (UEM). Núcleo de Pesquisa em Biotecnologia, Saúde e Meio Ambiente-NUPBSAM. E-mail: anpapegenpes@hotmail.com

${ }^{4}$ Professora do curso de Ciências Biológicas da Universidade Federal do Piauí, Picos, PI. Doutora em Genética e Melhoramento Vegetal pela Universidade Estadual de Maringá (UEM). Núcleo de Pesquisa em Biotecnologia, Saúde e Meio Ambiente (NUPBSAM). E-mail: smeireles@ufpi.edu.br (autora para contato) 
Resumo: O papel das macrófitas nos ecossistemas aquáticos pode ser avaliado a partir da determinação da sua biomassa, obtendo-se informações relacionadas ao tempo de crescimento, armazenamento de nutrientes, fluxos de energia e realocação de nutrientes. Os objetivos deste estudo foram identificar as espécies de macrófitas aquáticas em duas áreas de coletas no rio Guaribas e verificar as diferenças de biomassa das espécies encontradas para essas áreas. Foram definidos dois locais de amostragem ao longo do rio, na zona rural e urbana da cidade. As plantas que se encontravam dentro do quadrado foram coletadas com a mão, e as submersas com um gancho com pontas de ferro, colocadas em sacos plásticos devidamente identificados e levadas ao laboratório para identificação e pesagem da biomassa. Foram registradas nove espécies e Eichhornia crassipes e Salvinia auriculata apresentaram os maiores valores de peso seco, para a zona urbana e Chara cf. guairensis para a zona rural.

Palavras-chave: plantas aquáticas; alocação de recursos; produtividade; biomassa.

Abstract: The role of macrophytes in aquatic ecosystems can be evaluated by determining their biomass, obtaining information related to growth time, nutrient storage, energy fluxes and nutrient relocation. This study objectives were to identify the species of aquatic macrophytes in two areas of collections in the river Guaribas and to verify the biomass differences of the species found for these areas. Two sampling sites were defined along the river, in the rural and urban area of the city. The plants that were inside the square were collected by hand, and the submerged by a hook with iron tips and placed in plastic bags, properly identified and taken to the laboratory for identification and weighing of the biomass. Nine species were recorded and Eichhornia crassipes and Salvinia auriculata presented the highest dry weight values for the urban zone and Chara cf. Guairensis for rural areas.

Key words: aquatic plants; resource allocation; productivity; biomass.

Resumen: El papel de los macrófitos en los ecosistemas acuáticos se puede evaluar a partir de la determinación de su biomasa, la obtención de información relacionada con el tiempo de crecimiento, el almacenamiento de nutrientes, los flujos de energía y la reubicación de nutrientes. Los objetivos fueron identificar las especies de malas hierbas acuáticas en dos locales de colecciones en el río Guaribas y comprobar las diferencias en la biomasa de las especies que se encuentran en estas áreas. Se definieron dos puntos de muestreo a lo largo del río, en la zona rural y urbana. Las plantas que se encontraban en la plaza se recogieron a mano y la submergido con un gancho de hierro con puntas y se colocaron en bolsas de plástico, debidamente identificados y llevados al laboratorio para su identificación y pesaje de la biomasa. Nueve especies fueron registradas y Eichhornia crassipes y Salvinia auriculata mostraron los mayores valores de peso seco para el área urbana y Chara cf. guairensis al campo.

Palabras clave: las plantas acuáticas; asignación de recursos; la productividad; la biomasa. 


\section{INTRODUÇÃO}

As macrófitas aquáticas são plantas essenciais ao perfeito equilíbrio dos ambientes aquáticos, dando sustentabilidade a um elevado número de organismo, diminuindo a turbulência das águas e sedimentando os materiais em suspensão, principalmente em locais onde a mata ciliar foi suprimida (MOURA; FRANCO; MATALLO, 2009). Apresentam grande capacidade de adaptação e amplitude ecológica, habitando ambientes variados, desde brejos até ambientes verdadeiramente aquáticos, de água doce, salobra e salgada, ambientes de água estacionária e corrente. Incluem, portanto, desde macroalgas até plantas vasculares (MOURA; FRANCO; MATALLO, 2009). A capacidade que essas plantas possuem para colonizarem ambientes aquáticos, com diferentes características físico-químicas, está relacionada com as adaptações morfofisiológicas que elas detêm (THOMAZ et al., 1999).

Diferentes fatores abióticos dizem respeito à colonização e produtividade das espécies de macrófitas. Espécies submersas têm seu desenvolvimento e colonização afetados principalmente pela radiação subaquática e temperatura da água, enquanto as flutuantes são em geral restringidas por nutrientes e velocidade da corrente (PIERINI; THOMAZ, 2004; THOMAZ, 2005). Essa eficiência em aumentar sua densidade nos ambientes aquáticos, pode impossibilitar as atividades pesqueiras, de navegação, de captação de água e de lazer, além de provocar o entupimento de canais e danificar tubulações em reservatórios hidroelétricos (PAGIORO et al., 2005; HENRY-SILVA; CAMARGO, 2008).

O papel das macrófitas nos ecossistemas aquáticos pode ser avaliado a partir da determinação da sua biomassa, de onde podem ser obtidas informações relacionadas ao tempo de crescimento, armazenamento de nutrientes, fluxos de energia e realocação de nutrientes (THOMAS; BINI; PAGIORO, 2004). 
As ações antrópicas nos corpos d'água resultam em modificações das características abióticas dos ambientes aquáticos, provocando a depreciação da quantidade e qualidade da água dos mananciais e o comprometimento da fauna e flora a eles associadas (ESTEVES; BARBOSA, 1986). As adições de nutrientes, através de lançamentos de efluentes domésticos e industriais, ou por drenagens de áreas agrícolas, permitem o aumento de substâncias tais como fosfato, amônia e nitrato, que, em geral, são encontradas em baixas concentrações nos ambientes aquáticos. Como consequência, o aumento da disponibilidade de nutrientes provoca desequilíbrio, acelerando assim o crescimento da vegetação aquática indesejável (ESTEVES, 2011).

O Estado do Piauí, detentor de um elevado potencial hídrico, tem um número significativo de represas e barragens em seus rios, sejam eles perenes ou temporários. No município de Picos, o rio Guaribas teve o seu fluxo modificado em função da criação do reservatório de Bocaina, o que vem causando sérios problemas ambientais a esse ecossistema. Esses impactos estão contribuindo para que ocorram alterações no processo de estabelecimento das plantas aquáticas ali existentes, principalmente na zona urbana da cidade, região em que o rio está sujeito a um maior aporte de ações antrópicas, que podem contribuir de forma negativa para o seu funcionamento. Pouco se conhece desse ecossistema, principalmente em relação à diversidade de plantas que habitam suas águas. Portanto este estudo teve como objetivos identificar as espécies de macrófitas aquáticas em duas áreas de coletas no rio Guaribas e verificar as diferenças de biomassa das espécies encontradas para essas áreas. 


\section{MATERIAIS E MÉTODOS}

\section{1 Área de estudo}

O município de Picos, está localizado na região centro-sul do Estado do Piauí, a uma latitude de $07^{\circ} 04^{\prime} 37^{\prime \prime}$ S e $41^{\circ} 28^{\prime} 01^{\prime \prime}$ W, limitando-se ao norte com Santana do Piauí, ao sul com Itainópolis, a leste com Geminiano e Sussuapara, e a Oeste com Paquetá, Dom Expedito Lopes e Santa Cruz do Piauí. A população do município é de 73.414 habitantes (INSTITUTO BRASILEIRO DE GEOGRAFIA E ESTATÍSTICA [IBGE], 2010).

Na região predomina o clima tropical semiárido quente, apresentando uma temperatura média de $30^{\circ} \mathrm{C}$, ocorrendo um período seco de sete a oito meses por ano. Essas condições favorecem a predominância de plantas características dos biomas Caatinga e Cerrado, mas também ocorre o desenvolvimento de manchas de matas de cocais, onde predominam os carnaubais, caracterizando a região como um ecótono desses biomas.

A bacia hidrográfica do rio Guaribas está localizada na mesorregião sudeste do estado do Piauí, percorrendo 18 municípios, entre estes o município de Picos, o que corresponde a aproximadamente 3,35\% da área total do Estado. O Rio Guaribas é um rio típico de regiões semiáridas, apresentando descargas violentas e de curta duração na época de chuvas. As águas do Rio Guaribas são utilizadas para irrigação, exceto nas áreas próximas à cidade de Picos, onde o rio se encontra poluído e bastante assoreado, em função do desmatamento nas Áreas de Preservação Permanente.

\subsection{Método de coleta}

As coletas foram mensais e realizadas nos meses de fevereiro e março de 2015. Foram definidos dois locais de amostragem ao longo 
do rio Guaribas, um na zona rural e outro no centro da cidade. Em cada um desses locais, foram definidos três transectos, no sentido da margem para o leito do rio, distando $15 \mathrm{~m}$ entre si. As macrófitas foram coletadas com o auxílio de um quadrado feito de PVC com 0,25 $\mathrm{m}^{2}$. Em cada transecto, o quadrado foi lançado por três vezes em cada coleta, totalizando 18 coletas em cada local de amostragem e 36 coletas no total. As macrófitas aquáticas que se encontravam dentro do quadrado foram coletadas com a mão e com um gancho com pontas de ferro, para retirar as espécies submersas. Após a coleta, as amostras foram colocadas em sacos plásticos, devidamente identificados com o número de cada transecto, o ponto de coleta e levadas para o laboratório de Botânica e Limnologia da UFPI/Picos. No laboratório, o material foi separado e lavado para retirada de sedimentos, microalgas e outros materiais particulados que estivessem presos a elas. Após a lavagem, o material foi colocado em folhas de jornais, devidamente identificadas, para uma secagem parcial das plantas. A identificação taxonômica foi feita com auxílio da literatura especializada e por especialistas da área. Após a identificação, as amostras foram colocadas em sacos de papel devidamente identificados e levados à estufa de campo para secagem.

Diariamente as plantas foram pesadas em balança digital até atingir peso constante e a biomassa expressa em gramas de peso seco por metro quadrado (g.PS $\left./ \mathrm{m}^{2}\right)$.

\section{RESULTADOS E DISCUSSÃO}

Considerando os locais amostrados foram registradas nove espécies de macrófitas aquáticas, pertencentes a nove famílias (Tab. 1). 
Tabela 1- Lista de famílias e espécies de macrófitas aquáticas coletadas no rio Guaribas com indicação do local de coleta

\begin{tabular}{|l|l|l|}
\hline \multicolumn{1}{|c|}{ Família } & \multicolumn{1}{c|}{ Espécie } & \multicolumn{1}{c|}{ Local } \\
\hline Araceae & Pistia stratiotes L. & Zona rural \\
\hline Araliaceae & Hydrocotyle bonariensis Lam. & Centro \\
\hline Charophyceae & Chara cf. guairensis R.M.T. Bicudo & Zona rural \\
\hline Commelinaceae & Commelina sp & Centro \\
\hline Fabaceae & Neptunia oleracea Lour. & Zona rural \\
\hline Onagraceae & Ludwigia helmintorrhiza (Mart.) Hara & Zona rural \\
\hline Pontederiaceae & Eichhornia crassipes (Mart.) Solms & Centro \\
\hline Poaceae & Hymenachne amplexicaulis (Rudge) Nees & Rural \\
\hline Salvinaceae & Salvinia auriculata Aubl. & $\begin{array}{l}\text { Zona rural/ } \\
\text { Centro }\end{array}$ \\
\hline
\end{tabular}

Os resultados de biomassa obtidos para as coletas da zona urbana (Tabela 2) mostram que as espécies Eichhornia crassipes e Salvinia auriculata apresentaram os maiores valores de peso seco, enquanto Commelina sp o menor valor de peso seco.

Tabela 2 - Biomassa das espécies coletadas no rio Guaribas, zona urbana da cidade de Picos-Piauí

\begin{tabular}{|l|l|}
\hline Espécie & \multicolumn{1}{c|}{ Biomassa } \\
\hline Eichhornia crassipes & $387,71 \mathrm{~g} / \mathrm{PS} / \mathrm{m}^{2}$ \\
\hline Commelina sp & $5,93 \mathrm{~g} / \mathrm{PS} / \mathrm{m}^{2}$ \\
\hline Hydrocotyle bonariensis & $49,89 \mathrm{~g} / \mathrm{PS} / \mathrm{m}^{2}$ \\
\hline Salvinia auriculata & $104,81 \mathrm{~g} / \mathrm{PS} / \mathrm{m}^{2}$ \\
\hline
\end{tabular}

Para a zona rural, os resultados mostram que Chara cf. guairensis se destaca em valor de biomassa (Tabela 3), em relação às outras espécies identificadas para a área. 
Tabela 3- Biomassa das espécies coletadas no rio Guaribas, na zona rural da cidade de Picos-Piauí

\begin{tabular}{|l|l|}
\hline Espécie & Biomassa \\
\hline Chara cf. guairensis & $243,75 \mathrm{~g} / \mathrm{PS} / \mathrm{m}^{2}$ \\
\hline Hymenachne amplexicaulis & $23,15 \mathrm{~g} / \mathrm{PS} / \mathrm{m}^{2}$ \\
\hline Ludwigia helmintorrhiza & $23,51 \mathrm{~g} / \mathrm{PS} / \mathrm{m}^{2}$ \\
\hline Neptunia oleracea & $53,1 \mathrm{~g} / \mathrm{PS} / \mathrm{m}^{2}$ \\
\hline Pistia stratiotes & $0,08 \mathrm{~g} / \mathrm{PS} / \mathrm{m}^{2}$ \\
\hline Salvinia auriculata & $13,63 \mathrm{~g} / \mathrm{PS} / \mathrm{m}^{2}$ \\
\hline
\end{tabular}

A flora identificada para as áreas de coleta compõe-se de espécies características de ambientes com elevados teores de nutrientes, como por exemplo, Salvinia auriculata, pioneira na sucessão em locais perturbados, Eichhornia crassipes, que se caracteriza como uma das plantas daninhas mais agressivas do mundo, presente em reservatórios e lagos (MARTINS et al., 2009) e Pistia stratiotes, cujos maiores prejuízos, no Brasil, decorrentes de sua proliferação são causados nas áreas mais quentes e em mananciais poluídos (LORENZI, 2000). Portanto as alterações antropogênicas que vêm ocorrendo no rio Guaribas, tais como a eutrofização, podem promover o crescimento de $P$. stratiotes e provocar a expansão de sua área de distribuição. Essas plantas a presentam enorme potencial de propagação, podendo aumentar sua área de cobertura em $15 \%$ ao dia, dobrando-a a cada seis ou sete dias (MARTINS et al., 2009).

As espécies identificadas para o rio Guaribas refletem as condições em que o rio se encontra atualmente, após um longo período de estiagem, com pontos de água estagnada, extensas áreas de lamaçal, lançamento de nutrientes a partir de esgotos residenciais, comerciais e hospitalares, bem como de atividades de hortifruticulturas desenvolvidas em suas margens, o que aumenta o aporte de nutrientes lançados no leito do rio, favorecendo o estabelecimento dessas es- 
pécies de plantas aquáticas. A retirada da vegetação ciliar, para essas atividades, está contribuindo com o arraste do solo marginal para o leito do rio e diminuindo sua profundidade em certos locais, formando lagoas de águas claras e calmas, na zona rural, onde a concentração de nutrientes é menor e, na zona urbana, se formam verdadeiras lagoas de esgoto ou lama, onde predominam espécies como E. crassipes, H. bonariensis, S. auriculata e Commelina sp (Tabela 1). Tanto S. auriculata como E. crassipes são consideradas espécies indicadoras de ambientes eutrofizados (POTT; POTT, 2000), o que pode justificar a abundância dessas espécies na zona urbana.

No que se refere ao teor de biomassa (Tabela 2), E. crassipes é um vegetal que geralmente desenvolve raízes longas, com isso, é mais eficiente em explorar regiões mais profundas dos ecossistemas aquáticos, o que facilita a retirada de nutrientes do substrato e the dá maior capacidade de proliferação. Essa planta também tem, como característica, intensa capacidade de fixar em seus tecidos nutrientes em quantidades superiores às suas necessidades, além de elementos químicos estranhos a sua nutrição, portanto considerada uma planta atenuadora do processo de eutrofização (REIDEL et al., 2003). Esse fato pode justificar o maior valor de biomassa para a espécie, pois, na área urbana, onde foi coletada, o rio recebe um elevado aporte de matéria orgânica. A produção de E. crassipes pode chegar a $800 \mathrm{~kg} /$ ha/dia de matéria seca (LORENZI, 2008). Estudos revelam que essa espécie é capaz de apresentar elevada produção de biomassa, mesmo em condições de temperatura inferiores às favoráveis ao seu desenvolvimento pleno (WEIRICH et al., 2009).

Salvinia auriculata é uma espécie que ocorre com grande frequência em locais de água parada ou com pouco deslocamento, estado esse em que se encontra atualmente esse trecho do rio Guaribas. A S. auriculata cobre toda a superfícies da água nos ecossistemas aquáticos onde se estabelece, interrompendo a entrada de radiação 
solar, interferindo no ambiente e dificultando o desenvolvimento de outras espécies de plantas aquáticas, em especial as submersas, com isso, aumenta a sua produção de biomassa (MOURA et al., 2009). Estudos mostram que seus representantes priorizam o crescimento a partir da produção de clones do que por reprodução sexual, quando em baixas densidades e em condições de grande disponibilidade de água (WOLFF et al., 2012). Representantes do gênero Salvinia são considerados como uma das plantas daninhas aquáticas mais importantes, podendo chegar a uma produtividade de 110 toneladas/ha/ ano. Considerando o crescimento, biomassa, o número dos ramos pode dobrar em apenas três dias (MOURA et al., 2009).

Chara cf. guairensis se destacou no teor de biomassa, para a área de coleta da zona rural (Tabela 3). Representantes do gênero Chara geralmente habitam regiões de águas paradas e límpidas, com nível médio ou baixo em nutrientes, com temperatura mais elevada, menor velocidade de correnteza, formando estandes com elevada biomassa (VIEIRA JÚNIOR et al., 2003, HENRY-SILVA et al., 2013). Características condizentes com o local onde foi realizada a coleta dessa espécie, o que pode justificar sua abundância na área. Estandes dessas plantas que apresentam elevada biomassa são ecologicamente importantes na estruturação dos ambientes aquáticos, pois podem atuar na absorção e ciclagem de nitrogênio, principalmente por apresentarem altas taxas de produção primária e revestimento de determinados compostos, como calcita, que favorecem a retirada de nutrientes (HENRY-SILVA et al., 2013).

Neptunia oleracea é uma erva flutuante ou prostrada que, assim como Ludwigia helmintorrhiza, também flutuante, se distribui próximo à margem dos cursos d'água, nas zonas rasas de diferentes corpos d'água, essas espécies apresentam crescimento rápido o que exige muito espaço e têm seu desenvolvimento reduzido quando o nível da água baixa (POTT; POTT, 2000). Essas necessidades podem 
estar limitando o estabelecimento dessas espécies na zona urbana da cidade, por apresentar um estágio mais avançado de eutrofização.

Um fato que chama atenção é a $P$. stratiotes, espécie considerada indicadora de ambiente poluído, ter sido encontrada na zona rural, embora com valor muito baixo de biomassa, e não ser encontrada na zona urbana, local mais favorável ao seu estabelecimento. Isso pode indicar que, apesar de apresentar água com aparência límpida, o rio na zona rural já tem suas águas comprometidas. A ausência de $P$. stratiotes na zona urbana pode ser explicada pela presença de $S$. auriculata e E. crassipes, pois é de conhecimento que $P$. stratiotes é um fraco concorrente de outras espécies flutuantes (BOTTINO; CALIJURI; MURPHY, 2013).

Pesquisas relacionadas à produtividade primária de espécies de macrófitas aquáticas, de diversos grupos ecológicos, têm identificado uma enorme variedade interespecífica, sendo que as espécies emersas e flutuantes são as que conferem os maiores índices de produtividade, fundamentalmente quando confrontadas com aqueles obtidos para as macrófitas aquáticas submersas e com folhas flutuantes (ESTEVES, 2011). Esses dados estão de acordo com os que foram encontrados para E. crassipes e S. auriculata (flutuantes), no entanto diferem no que se refere à Chara cf. guairensis, uma macrófita submersa, que apresentou valor de biomassa equivalente ao apresentado pelas espécies flutuantes. Esses dados são importantes e chamam atenção para a necessidade de estudos nessa região, visto que esta é detentora de um número elevado de afloramentos hídricos, onde são encontradas diversas espécies de macrófitas aquáticas e que, ao mesmo tempo, estão sobre forte pressão antrópica, o que pode resultar na diminuição ou eliminação dessas plantas. Espera-se que este trabalho possa servir de suporte para outras pesquisas que possam ampliar o conhecimento sobre o papel que esses organismos desempenham nos ecossistemas hídricos, bem como de subsídio para que o poder 
público possa realizar ações de fiscalização e controle de uso desses recursos hídricos e também desenvolver projetos de recuperação e revitalização do rio Guaribas, um dos ambientes aquáticos da região, com maior sobrecarga de impactos antrópicos negativos.

\section{REFERÊNCIAS}

BOTTINO, F.; CALIJURI, M. do C.; MURPHY, K. J. Temporal and spatial variation of limnological variables and biomass of different macrophyte species in a Neotropical reservoir (São Paulo - Brazil). Acta Limnologica Brasiliensia, v. 25, n. 4, p. 387-397, 2013. DOI http://dx.doi.org/10.1590/ S2179-975X2013000400004

ESTEVES, F. A. Fundamentos de limnologia. 3. ed. Rio de Janeiro: Interciência, 2011.

ESTEVES, F. A.; BARBOSA, F. A. R. Eutrofização artificial: a doença dos lagos. Ciência Hoje, v. 5, n. 27, p. 56-61, 1986.

HENRY-SILVA, G. G.; CAMARGO, A. F. M. Impacto das atividades de aquicultura e sistemas de tratamento de efluentes com macrófitas aquáticas - relato de caso. Boletim do Instituto de Pesca, v. 34, n. 1, p. 163-173, 2008.

HENRY-SILVA, G. G.; SANTOS, R. V.; MOURA, R. S. T. ; BUENO, N. C. Primeiro registro de Chara indica e Chara zeylanica (Charophyceae, Charales, Characeae) em reservatórios do semiárido do estado do Rio Grande do Norte, Brasil. Biotemas, v. 26, n. 3, p. 243-248, 2013. DOI: http://dx.doi. org/10.5007/2175-7925

INSTITUTO BRASILEIRO DE GEOGRAFIA E ESTATÍ́sTICA (IBGE). Censo demográfico 2010. Rio de Janeiro, 2010.

LORENZI, H. Plantas daninhas do Brasil: terrestres, aquáticas, parasitas e tóxicas. Nova Odessa, SP: Instituto Plantarum, 2008.

. Plantas daninhas do Brasil: terrestre, aquáticas, parasitas, tóxicas e medicinais. Nova Odessa, SP: Instituto Plantarum, 2000.

MARTINS, D.; CARBONARI, C. A.; TERRA, M. A.; MARCHI, S. R. Ação de adjuvantes na absorção e translocação de glyphosate em plantas de aguapé (Eichhornia crassipes). Planta Daninha, v. 27, n. 1, p. 155-163, 2009. 
MOURA, M. A. M.; FRANCO, D. A. S.; MATALLO, M. B. Manejo integrado de macrófitas aquáticas. Divulgação Técnica Biológico, São Paulo, v. 71, n. 1, p. 77-82, 2009.

PAGIORO, T. A.; ROBERTO, M. C.; THOMAZ, S. M.; PIERINI, S. A.; TAKA, M. Zonação longitudinal das variáveis limnológicas abióticas em reservatórios. In: RODRIGUES, L. (Org.). Biocenose em reservatórios: padrões espaciais e temporais. São Carlos, SP: RiMa, 2005. p. 39-46.

PIERINI, S. A.; THOMAZ, S. M. Adaptações de plantas submersas à absorção do carbono inorgânico. Acta Botanica Brasilica, v. 18, n. 3, p. 629-641, 2004. POTT, V. J.; POTT, A. Plantas aquáticas do Pantanal. Corumbá, MS: EMBRAPACPAP, 2000.

REIDEL, A.; GÜTHS, R.; FEIDEN, A.; DAMASCENO, S.; CUNHA, F.; SIGNOR, A. A. Produção de biomassa de aguapé (Eichhornia crassipes (Mart. Solms) fertilizados com diferentes adubos. Revista Varia Scientia, v. 3, n. 6, p. 95101, 2003.

THOMAZ, S. M.; BINI, L. M.; SOUZA, M. C.; KITA, K. K.; CAMARGO, A. F. M. Aquatic macrophytes of Itaipu reservoir, Brazil: survey of species and ecological considerations. Brazilian Archives of Biology and Technology, Curitiba, v. 42, n. 1, p. 15-22, 1999.

THOMAZ, S. M.; BINI, L. M. Análise crítica dos estudos sobre macrófitas aquáticas desenvolvidos no Brasil. In: THOMAZ, S. M.; BINI, L. M. (Ed.). Ecologia e manejo de macrófitas. Maringá, PR: EDUEM, 2003. p 19-38.

THOMAZ, S. M.; BINI, L. M.; PAGIORO, T. A. Métodos em limnologia: macrófitas aquáticas. In: BICUDO, C. E. M.; BICUDO, D. C. (Org.). Amostragem em limnologia. São Carlos, SP: RiMa, 2004. p. 193-210.

THOMAZ, S. M. Fatores que afetam a distribuição e o desenvolvimento de macrófitas aquáticas em reservatório: uma análise em diferentes escalas. In: NOGUEIRA, M. G., HENRY, R.; JORCIN, A. (Org.). Ecologia de reservatório: impactos potenciais, ações de manejo e sistemas em cascata. São Carlos, SP: RiMa, 2005. p. 165-181.

VIEIRA JÚNIOR, J.; NECCHI JÚNIOR, O. ; BRANCO, C. C. Z.; BRANCO, L. H. Z. Characeae (Chlorophyta) em ecossistemas lóticos do estado de São Paulo, Brasil: gênero Chara e distribuição ecológica. Hoehnea, v. 30, n. 1, p. 53-70, 2003. 
WEIRICH, C.; COSTA, J. M.; KLOZOVSKI, É. S.; FEIDEN, A.; BOSCOLO, W. R. Seasonal production of biomass in two species of aquatic macrophytes floating (Eichhornia crassipes e Pistia stratiotes) on effluent treatment systems. Revista Brasileira de Agroecologia, v. 4, n. 2, p. 2970-2973, 2009.

WOLFF, G.; PEREIRA, G. C.; CASTRO, E. M.; LOUZADA, J.; COELHO, F. F. The use of Salvinia auriculata as a bioindicator in aquatic ecosystems: biomass and structure dependent on the cadmium concentration. Brazilian Journal of Biology, v. 72, n. 1, p. 71-77, 2012. 